\title{
On the inorganic carriers of the 21 micron emission feature in post-AGB stars
}

\author{
Ke Zhang ${ }^{1}$, Biwei Jiang ${ }^{1}$, and Aigen $\mathrm{Li}^{2}$ \\ ${ }^{1}$ Department of Astronomy, Beijing Normal University, Beijing 100875, China \\ emails: zhangke@mail.bnu.edu.cn, bjiang@bnu.edu.cn \\ ${ }^{2}$ Department of Physics \& Astronomy, University of Missouri, Columbia, MO 65211, USA \\ email: lia@missouri.edu
}

\begin{abstract}
The mysterious $21 \mu \mathrm{m}$ emission feature seen in only $12 \mathrm{C}$-rich proto-planetary nebulae (PPNe) remains unidentified since its discovery in 1989. Over a dozen materials have been suggested as the carrier candidates while none of them has received general acceptance. We investigate the inorganic carrier candidates by applying the observational constraints of the feature strength and associated features. It is found that: (1) three candidates, TiC clusters, fullerenes with $\mathrm{Ti}$ impurity atoms, and $\mathrm{SiS}_{2}$, are not abundant enough to account for the emission power of the $21 \mu \mathrm{m}$ band, (2) five candidates, doped-SiC, $\mathrm{SiO}_{2}$-mantled $\mathrm{SiC}$ dust, carbon and silicon mixtures, $\mathrm{Fe}_{2} \mathrm{O}_{3}$, and $\mathrm{Fe}_{3} \mathrm{O}_{4}$, all show associated features which are either not detected in the $21 \mu \mathrm{m}$ sources or detected but with a much lower strength, and (3) FeO, which satisfies the abundance constraints, does not display any associated features which are not seen in the $21 \mu \mathrm{m}$ sources. Moreover, $\mathrm{FeO}$ is more likely to survive in the C-rich environment than $\mathrm{Fe}_{2} \mathrm{O}_{3}$ and $\mathrm{Fe}_{3} \mathrm{O}_{4}$. Thus $\mathrm{FeO}$ seems to be the most plausible one among the inorganic carrier candidates.
\end{abstract}

Keywords. Infrared: stars, (stars:) circumstellar matter, stars: AGB and post-AGB

\section{Introduction}

The so-called " $21 \mu \mathrm{m}$ feature" has been well identified in 12 proto-planetary nebulae (PPNe; Kwok et al. 1999; also see Hrivnak et al. in this proceeding for two new $21 \mu \mathrm{m}$ sources). This feature, peaking at $\sim 20.1 \mu \mathrm{m}$ with a FWHM of $\sim 2.2-2.3 \mu \mathrm{m}$, has little shape variation among different sources (Volk et al. 1999). Most of these sources exhibit quite uniform characteristics: metal-poor, carbon-rich F and G supergiants with strong infrared (IR) excess and over abundant s-process elements (see Kwok et al. 1999 and Zhang et al. 2006).

Since its discovery by Kwok et al. (1989), over a dozen of both organic and inorganic carrier candidates have been proposed. The inorganic candidates are: (a) TiC nanoclusters (von Helden et al. 2000, but see Li 2003), (b) large-cage carbon particles (fullerenes) coordinated with Ti atoms (Kimura et al. 2005), (c) $\mathrm{SiS}_{2}$ dust (Goebel 1993), (d) SiC dust with carbon impurities (Speck \& Hofmeister 2004, but see Jiang et al. 2005), (e) carbon and silicon mixtures (Kimura et al. 2005), (f) $\mathrm{SiC}_{\text {core- }} \mathrm{SiO}_{2}$ mantle grains (Posch et al. 2004), (g) $\mathrm{FeO}$ (Posch et al. 2004), (h) $\mathrm{Fe}_{2} \mathrm{O}_{3}$, and (i) $\mathrm{Fe}_{3} \mathrm{O}_{4}$ (Cox 1990). However, none of these carrier candidates have received general acceptance. The carrier of the $21 \mu \mathrm{m}$ feature remains unidentified. In this paper we report our recent efforts in testing the above-listed inorganic candidates in terms of the elemental abundance budget and spectral shape (with special emphasis on the possible accompanying features).

\section{General Constraints: Band Strength and Associated Features}

We assess the applicability of a proposed carrier candidate by examining (1) whether it is capable of emitting the observed large amount of energy in the $21 \mu \mathrm{m}$ band without 
requiring more dust material than available, and (2) whether the candidate carrier produces additional feature(s) with intensities inconsistent with those observed. HD 56126, a prototypical $21 \mu \mathrm{m}$ feature source, is taken as the test case object.

The total energy emitted in the $21 \mu \mathrm{m}$ band $E_{\text {tot }}$ is related to the abundance (relative to $\mathrm{H}$ ) of element $\mathrm{X}$ of the carrier by $[\mathrm{X} / \mathrm{H}]=\frac{n_{\mathrm{X}} E_{\mathrm{tot}}}{\mu_{\mathrm{d}} M_{\mathrm{H}} \int_{21 \mu \mathrm{m} \mathrm{band}} \kappa_{\mathrm{abs}}(\lambda) \times 4 \pi B_{\lambda}\left(T_{\mathrm{d}}\right) d \lambda}$ (see Zhang et al. 2008). Knowing the dust temperature $T_{\mathrm{d}}$ and the mass absorption coefficient $\kappa_{\mathrm{abs}}$, one can calculate the element abundance required for a dust species (containing $n_{\mathrm{X}} \mathrm{X}$ atoms in each molecule with a molecular weight $\mu_{\mathrm{d}}$ ) to account for the observed intensity of the $21 \mu \mathrm{m}$ band. This constraint effectively excludes those candidates with scarce elements such as Ti. On the other hand, some of the proposed carrier candidates exhibit strong features in addition to the $21 \mu \mathrm{m}$ band which are not seen in the $21 \mu \mathrm{m}$ sources. Using lab optical constants and the stellar parameters, we calculate the intensity ratios of the associated features to the $21 \mu \mathrm{m}$ band and compare with the observed spectra. In this way we exclude most of the candidates which display associated feature(s), such as SiC-bearing dust.

\section{Results}

Our results on individual carrier candidate are shown in Table 1. We investigate nine inorganic carrier candidates. Three of them are excluded due to abundance deficiency and another five suffer from producing strong associated features not seen in the $21 \mu \mathrm{m}$ sources. At this moment, FeO nano particles seem to be the most promising candidate.

Table 1. Summary of the test on the carrier candidates $(\checkmark$ : pass; $\times$ : fail)

\begin{tabular}{ccc}
\hline Candidate & Abundance (element) & Associated features \\
\hline $\mathrm{TiC}$ nanoclusters & $\times(\mathrm{Ti})$ & $\checkmark$ \\
\hline fullerenes coordinated with Ti atoms & $\times(\mathrm{Ti})$ & $\checkmark$ \\
\hline $\mathrm{SiS}_{2}$ & $\times(\mathrm{S})$ & $\times(16.8 \mu \mathrm{m})$ \\
\hline $\mathrm{SiC}$ dust with carbon impurities & $\checkmark$ & $\times(11.3 \mu \mathrm{m})$ \\
\hline carbon and silicon mixtures & $\checkmark$ & $\times(8.3 \mu \mathrm{m}, 11.3 \mu \mathrm{m})$ \\
\hline $\mathrm{SiC}$ core-SiO \\
2 \\
\hline $\mathrm{FeO}$ antle grains & $\checkmark$ & $\checkmark$ \\
\hline $\mathrm{Fe}_{2} \mathrm{O}_{3}$ & $\checkmark$ & $\times(9.2,18,27.5 \mu \mathrm{m})$ \\
\hline $\mathrm{Fe}_{3} \mathrm{O}_{4}$ & $\checkmark$ & $\times(16.5,24 \mu \mathrm{m})$ \\
\hline
\end{tabular}

\section{References}

Cox, P. 1990, A\&A (Letters), 236, L29

Goebel, J. H. 1993, A\&A, 278, 226

Jiang, B. W., Zhang, K., \& Li, A. 2005, ApJ (Letters), 630, L77

Kimura, Y., Nuth, J. A., III, \& Ferguson, F. T. 2005, ApJ (Letters), 632, L159

Kwok, S., Volk, K., \& Hrivnak, B. J. 1999, in Asymptotic Giant Branch Stars, p. 297

Kwok, S., Volk, K. M., \& Hrivnak, B. J. 1989, ApJ, 345, 51

Li, A. 2003, ApJ (Letters), 599, L45

Posch, T., Mutschke, H., \& Andersen, A. 2004, ApJ, 616, 1167

Speck, A. K., \& Hofmeister, A. M. 2004, ApJ, 600, 986

Volk, K., Kwok, S., \& Hrivnak, B. J. 1999, ApJ (Letters), 516, L99

von Helden, G., Tielens, A. G. G. M., van Heijnsbergen, D. et al. 2000, Science, 288, 313

Zhang, K., Jiang, B. W., \& Li, A. 2006, Progress in Astronomy (Chinese), 24, 43

Zhang, K., Jiang, B. W., \& Li, A. 2008, in preparation 